\section{REVIEW OF MEDICATION DIRECTIVES IN DISCHARGES FROM ACUTE HOSPITAL TO COMMUNITY PALLIATIVE CARE SETTING}

Jennifer Brennock, Aisling O' Gorman, Maeve Hennessy, Grainne Tipping. Our Lady of Lourdes Hospital, Drogheda

\subsection{6/bmjspcare-2019-ASP.86}

Background Medication directives are used by Community Palliative Care Teams (CPCT) to allow planned, safe administration of medications by teams in the community setting for patients with chronic, life-limiting conditions for symptom control (SC) or end of life care (EOLC). This often allows patients to remain in the community and receive care that they may otherwise have had to receive in an in-patient setting.

Aims The aims of this project were to assess:

- Number of patients who were discharged with a medications directive from an acute hospital setting to CPCT;

- Duration of care under CPCT;

- Circumstances of any re-admission to acute hospital following discharge with medication directive.

Method Review of discharged in-patients to CPCT from Jan Dec 2017 using hospital palliative care team (HPCT) and CPCT databases and medication directive records, hospital electronic database and patient charts. Review was conducted by one person over a two week period and results were tabulated and analysed using simple Excel format.

Results 47 discharges to CPCT with medical directives were identified (38 EOLC, 9 SC). 2 were re-admitted following discharge with EOLC directive, and all 9 SC patients were readmitted (re-admission date range was 1-16 weeks postdischarge). 6 patients discharged with EOLC directive remained under CPCT care at time of review, 3 were discharged from service, with 32 dying under care of CPCT (median days after discharge 8.5).

Conclusion Medication directives helped to facilitate $84 \%$ of patients discharged from acute hospital to CPCT care to remain at home for EOLC. $100 \%$ of SC patients required readmission but had a varying period in the community prior to re-admission. This clearly demonstrates the importance of medication directives in both HPCT and CPCT for effective palliative care.

\section{THE QUALITY OF DOCUMENTATION IN THE CARE PLAN FOR END OF LIFE IN A DISTRICT GENERAL HOSPITAL: A QUALITY IMPROVEMENT PROJECT}

Despoina-Elvira Karakitsiou, Kathryn Gaunt, Yvette Heatley. Clatterbridge Cancer Centre, East Cheshire NHS Trust, East Cheshire NHS Trust/End of Life Partnership

10.1136/bmjspcare-2019-ASP.87

Quality Statement 8 of NICE Quality Standards 'End of Life Care for Adults' (2017) highlights the importance of coordination of care between different healthcare professionals.

In Macclesfield District General Hospital, a patient's end of life care is documented in a specific Care Plan for end of life, containing 7 different sections. In order to ensure that all health and social care professionals are aware of patients' individualised plans of care at the end of life, daily reviews by all HCPs should be documented within section 6 (Individualised care and daily nurse review) of the EOL care plan.
A baseline audit of documentation within the care plan for one month noted a lack of consistency in the location of HCP documentation. Daily reviews could be documented in the medical notes, nursing notes or within the care plan. This was felt to negatively impact upon the coordination of care for dying patients within the hospital.

In order to improve the consistency of documentation within section 6 of the care plan a sticker was introduced with the same colour as the care plan into medical and nursing notes when a patient commenced on an end of life care plan, which directed all HCPs to document within section 6 of the care plan.

A re-audit of the notes of all deaths on a care plan over one month was carried out following this intervention. In total the care plan was used for 12 patients and the sticker used in 8 of those deaths (66\%) Documentation within section 6 of the care plan improved with the use of the sticker, however, within the hospital the use of the care plan in general is inconsistent. This QIP has highlighted the need for review of EOL documentation and ongoing education and training.

\section{PATIENTS' AND CARERS' PERSPECTIVES ON THE USE OF THE PROGNOSIS IN PALLIATIVE CARE SCALES (PIPS) PREDICTOR MODELS: A QUALITATIVE STUDY}

Karen Spencer, Anastasia Kalpakidou, Patrick Stone, Chris Todd, Vaughan Keeley, Rumana Omar, Victoria Vickerstaff, Dori-Anne Finlay, Peter Buckle, Jane Griffiths. The University of Manchester, University College London, Derby Teaching Hospitals NHS Foundation Trust

\subsection{6/bmjspcare-2019-ASP.88}

Background Patients with advanced cancer and their carers often wish to know how long they have left to live, yet clinicians predictions of survival are often inaccurate and overoptimistic. The PiPS models use objective clinical data to predict whether palliative cancer patients will die within 'days', 'weeks' or 'months'. A previous study has shown that the PiPS models are at least as good as or more accurate than, clinicians'predictions of survival. We are currently validating the latter models in a large clinical study of palliative cancer patients $(n=1884)$, this paper presents data from a nested qualitative study that explored patients'with advanced cancer and their carers'perspectives of the PiPS predictor models.

Methods We recruited a purposive sample of patients $(n=29)$ and carers $(n=20)$ from a hospice inpatient unit and a hospital day care unit. Patients and carers were asked about their preferences for prognostic information, preferred way of communicating life expectancy and acceptability of the PiPS models.

Results The majority of participants expressed desire for detailed prognostic information. Participants'preferences were influenced by patient age, time since diagnosis and coping ability. Most agreed that having life expectancy presented as days, weeks or months was the most meaningful. All considered the PiPS models acceptable for use in clinical practice and a useful tool in helping clinicians more accurately predict life expectancy.

Conclusion This study has highlighted that palliative care patients and carers wish to discuss accurate prognostication with clinicians. Our study further demonstrates the acceptability of the PiPS models to palliative care patients and their carers. 